\title{
Another argument for embedded scalar implicatures based on oddness in downward entailing contexts*
}

\author{
Giorgio Magri \\ IJN, ENS
}

\begin{abstract}
In Magri 2009a,b, I argue that a sentence such as \#Some Italians come from a warm country sounds odd because it triggers the scalar implicature that not all Italians come from a warm country, which mismatches with the common knowledge that all Italians come from the same country. If this proposal is on the right track, then oddness can be used as a diagnostic for scalar implicatures. In this paper, I use this diagnostic to provide one more argument that scalar implicatures are computed not only at the matrix level but also in embedded position. The argument is based on a puzzling pattern of oddness in downward entailing contexts.
\end{abstract}

Keywords: scalar implicatures; downward entailing contexts; oddness

\section{Introduction}

The (a) sentences in (1)-(3) have an existential quantifier and sound odd (I use "\#" as a diacritic for oddness); the variants in (b) sound impeccable. This contrast is puzzling. Take for instance the pair in (1). We know that all Italians come from the same country. Thus, (1a) and (1b) convey the same information, namely that Italy is warm. Why is it then that only (1b) sounds fine?

a. \#Some Italians come from a warm country.

b. Italians come from a warm country.

(2) Context: in a society where children get the last name of their father.

a. \#Some of the children of that couple have a funny last name.

b. The children of that couple have a funny last name.

(3) Context: prof. Smith always assigns the same grade (possibly a different one every term) to all of his students. [E. Chemla, as p.c. in Schlenker 2006]

a. \#This year, prof. Smith assigned an A to some of his students.

b. This year, prof. Smith assigned an A to his students.

* Many thanks to Danny Fox. This work was supported in part by a 'Euryi' grant from the European Science Foundation ("Presupposition: A Formal Pragmatic Approach" - P. Schlenker). 
Another argument for embedded scalar implicatures

In general, an existentially quantified sentence such as (4a) triggers the scalar implicature that the corresponding universally quantified alternative (4b) is false.

(4) a. John did some of the homework.

b. John did all of the homework.

In Magri 2009a,b, I argued that this scalar implicature is the driving force of the oddness of (1a)-(3a). In (5), I illustrate the idea informally for the case of (1a). Let me dub (5) Hawkins' reasoning, as it is close to a proposal by Hawkins 1991; see Heim 1991, Sauerland 2008 and Percus 2006 for discussion.

(5) a. Because of existential quantification, (1a) triggers the scalar implicature that not all Italians come from a warm country.

b. But common knowledge entails that, if some Italians come from a warm country, then all of them do, because they come from the same country.

c. The oddness of (1a) thus follows from the mismatch between the implicature (5a) and common knowledge (5b).

The starting point of this paper is the conjecture that Hawkins' reasoning (5) is on the right track. Thus, oddness can be used as a diagnostic for scalar implicatures. An important recent debate concerns the issue of whether scalar implicatures can be computed in embedded positions. The tools used so far to detect alleged embedded implicatures comprise: introspective intuitions, as in Chierchia 2004, Russell 2006; experimental methods, as in Geurts \& Pouscoulous 2009, Chemla \& Spector 2009; and theoretical diagnostics, as in Chierchia, Fox \& Spector to appear. This paper contributes a new theoretical diagnostic: it argues that oddness can be used to detect implicatures in embedded positions. Actually, in surprising embedded positions, namely in downward entailing (DE) contexts. The paper is organized as follows. To set the stage, section 2 reviews the formalization of Hawkins' reasoning (5) that I have proposed in Magri 2009a,b. Section 3 then introduces a rather intriguing pattern of oddness in DE contexts, that has not been documented so far in the literature, to the best of my knowledge. Section 4 then argues that this pattern falls into place along the lines of my formalization of Hawkins' reasoning, as long as we posit embedded scalar implicatures. In the end, the paper thus presents a new theoretical argument in favor of embedded scalar implicatures.

\section{Assumptions on scalar implicatures}

Each of the three steps of Hawkins' reasoning (5) imposes a specific requirement on the underlying theory of scalar implicatures. Step (5a) requires the algorithm for the computation of scalar implicatures to be blind to common knowledge, otherwise the odd sentence (Some Italians come...) and its fine alternative (All Italians 
come...) would be indistinguishable and their different status left unexplained. Step (5b) requires common knowledge to kick in eventually, in order to derive the mismatch. Step (5c) requires the mismatching implicature to stay firmly in place against common knowldge, rather than being retracted and thus the odd sentence rescued from its oddness. In Magri 2009a,b, I discuss these desiderata in more detail, and I sketch a theory of scalar implicatures that meets these desiderata and thus formalizes Hawkins' reasoning (5). In this section, I review that proposal.

Background assumptions. I assume that scalar implicatures are brought about by a covert operator akin to overt 'only', called the exhaustivity operator and notated EXH. Just as it is the case for any overt quantifier, I assume that also the domain of this exhaustivity operator is restricted by a contextually assigned relevance predicate $\mathscr{R}$, so that I will write $\operatorname{EXH}_{\mathscr{R}}$. This operator $\operatorname{EXH}_{\mathscr{R}}$ has the semantics in (6). It takes a proposition $\varphi$, called the prejacent; and it does two things. First, it asserts the prejacent, as in (6a). Second, it negates every alternative $\psi$ to the prejacent that satisfies two requirements, as in (6b): $\psi$ is relevant, namely, it belongs to the relevance predicate $\mathscr{R}$; and $\psi$ is excludable, namely it belongs to the set of excludable alternatives $\mathscr{E} x c l(\varphi)$.

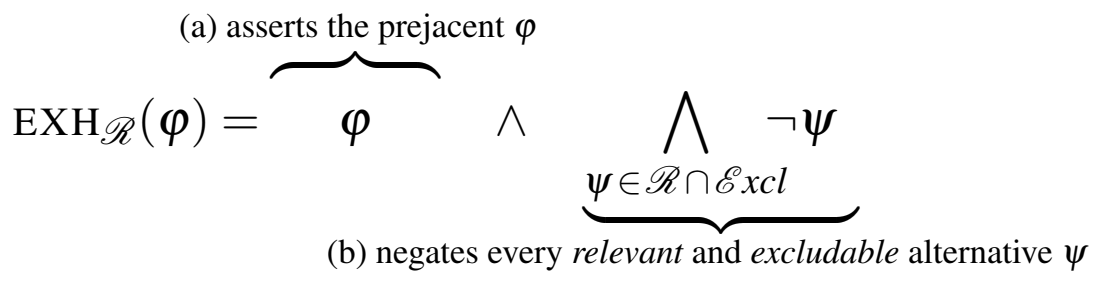

There are competing views on the proper definition of the set $\mathscr{E} x c l(\varphi)$ of alternatives $\psi$ excludable w.r.t. the prejacent $\varphi$. Two options are spelled out in (7). The choice between (7a) and (7b) matters (slightly); see footnote 7. I adopt the weaker (7b).

a. $\psi \in \mathscr{E} x c l(\varphi)$ iff $\psi$ asymmetrically entails $\varphi$.

b. $\psi \in \mathscr{E} x c l(\varphi)$ iff $\psi$ can be negated consistently with $\varphi$.

These background assumptions have been defended independently; see in particular Fox 2007, ${ }^{1}$ Chierchia et al. to appear and Fox \& Katzir 2009. On top of these background assumptions, I would like to add three more new(ish) assumptions.

New(ish) assumptions. When computing the relative strength of the prejacent $\varphi$ and one of its alternatives $\psi$ in order to establish whether (7) holds and $\psi$ is thus excludable, we have available two notions of entailment, spelled out in (8). ${ }^{2}$

1 In particular, assumption (7b) is a simplified version of a proposal in Fox 2007.

2 Of course, also (7b), just as (7a), can be restated in terms of entailment, by requiring that the negation of $\psi$ does not entail the negation of $\varphi$. 
Another argument for embedded scalar implicatures

a. $\psi$ logically entails $\varphi$ iff every $\psi$-world is a $\varphi$-world;

b. $\psi$ entails $\varphi$ given common knowledge iff every $\psi$-world compatible with common knowledge is a $\varphi$-world.

Which of these two notions of entailment (8) is the one relevant for the computation (7) of excludable alternatives? I submit that it is the notion of logical entailment, as stated in my first new(ish) assumption (9); see also Fox \& Hackl 2006.

(9) The computation of excludable alternatives is blind to common knowledge, in the sense that excludable alternatives are those alternatives $\psi$ that are logically consistent with the negation of the prejacent $\varphi$.

The relevance predicate $\mathscr{R}$, that restricts the domain of alternatives that the exhaustivity operator (6) quantifies over, is assigned by context. Yet, valid assignments must satisfy certain axioms. My second new(ish) assumption is two such axioms, stated in (10). Axiom (10a) might be related to Grice's (1975) "Maxim of Relevance". Axiom (10b) follows from the intuition that relevance is a contextual notion and is thus closed w.r.t. contextual equivalence.

(10) a. The prejacent of the exhaustivity operator is relevant.

b. If two propositions are contextually equivalent, then they pattern alike w.r.t. relevance, namely they are both relevant or else both irrelevant.

The exhaustivity operator is covert. Any covert category raises the recoverability problem: how do we recover whether the category is instantiated or not in a given LF? Let me suggest that there is not really any recoverability problem for the exhaustivity operator, because of (11), which is my third new(ish) assumption.

(11) The exhaustivity operator is syntactically mandatory at matrix scope.

To summarize, let me make explicit the correspondence between the three new(ish) assumptions (9)-(11) and the three desiderata associated with the three steps of Hawkins' reasoning (5). Step (5a) requires common knowledge to be kicked out of the computation of scalar implicatures, in order for the existential prejacent and the universal alternative to be distinguishable despite the fact that they are equivalent given common knowledge. This desideratum is met through assumption (9) that the set of excludable alternatives is computed blind to common knowledge. Yet, step (5b) requires common knowledge to kick back in eventually, in order to derive the mismatch between the implicature and common knowledge. This desideratum is met through assumption (10), that the computation of implicatures depends on relevance $\mathscr{R}$ which in turn is sensitive to common knowledge, through the closure property (10b). Finally, step (5c) requires the mismatching implicature to be firmly locked into place in order to enforce oddness. This desideratum is met by assumption (11) that the exhaustivity operator is syntactically mandatory. Let me turn to the details. 
Formalization of Hawkins' reasoning Let me now show how the three new(ish) assumptions (9)-(11) implement Hawkins' reasoning (5) for the oddness of (1a). By assumption (11), the LF of sentence (1a) is (12).

(12) $\left[\mathrm{EXH}_{\mathscr{R}}[\varphi\right.$ Some Italians come from a warm country $\left.]\right]$

The matrix prejacent $\varphi$ comes with a unique scalar alternative, namely $\psi$ in (13), obtained from the prejacent $\varphi$ by replacing some with the Horn-mate all.

(13) $\psi=$ All Italians come from a warm country.

The negation of this alternative $\psi$ is logically compatible with the prejacent $\varphi$, because of worlds such as (14), where the prejacent $\varphi$ is true but the alternative $\psi$ false, as some but not all of the Italians come from a warm country. Note that worlds such as (14) are not compatible with the piece of common knowledge that all Italians come from the same country. Thus, the negation of the alternative $\psi$ is not compatible with the prejacent $\varphi$ relative to common knowledge. (14)

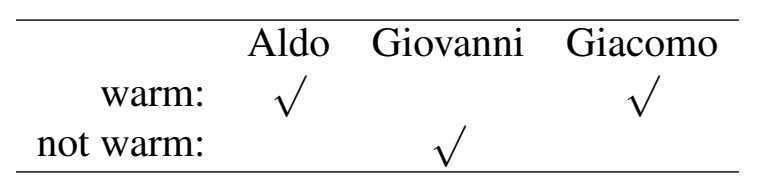

By the definition (6) of the exhaustivity operator together with the blindness assumption (9) that the computation of the set of excludable alternatives (7) is blind to common knowledge, the LF (12) gets interpreted as in (15).

$$
\begin{aligned}
{[[(12)]} & =\operatorname{EXH}_{\mathscr{R}}(\varphi) \\
& =\varphi \wedge(\neg \psi \vee \neg \mathscr{R}(\psi)) \\
& =\underbrace{\text { some Italians c.f.w.c. }}_{\varphi} \text { and }(\underbrace{\text { not all do }}_{\neg \psi} \text { or } \underbrace{\text { that is not relevant }}_{\neg \mathscr{R} \psi})
\end{aligned}
$$

By axiom (10a) on the contextually supplied relevance predicate $\mathscr{R}$, the prejacent $\varphi$ is relevant; by axiom (10b), the alternative $\psi$ is thus relevant too, because contextually equivalent to $\varphi$. Since $\mathscr{R}(\psi)$ is true, (15) can be simplified as in (16).

$$
[(12)]=\varphi \wedge \neg \psi=\underbrace{\text { Some Italians c.f.w.c. }}_{\varphi} \text { and } \underbrace{\text { not all do }}_{\neg \psi}
$$

The meaning derived in (16) is a contextual contradiction, given the piece of common knowledge that all Italians come from the same country. The oddness of sentence (1a) thus follows from the fact that it unambiguously denotes a contextual contradiction.

Nothing changes for plain cases Out of the blue, the alternative (17b) is stronger than the target (17a) w.r.t. both logic and contextual entailment. This I call a plain case. Before I close this section, let me make sure that the three new(ish) assumptions (9)-(11) do not mess around with plain cases. 
Another argument for embedded scalar implicatures

a. John did some of the homework.

b. John did all of the homework.

Assumption (9), that excludable alternatives are computed relative to logical entailment rather than contextual entailment, has no effect for plain cases, since the alternative (17b) is stronger than the prejacent (17a) no matter which one of the two entailments we pick. Also assumption (10b), that relevance is closed w.r.t. contextual equivalence, is mute for plain cases, since the prejacent (17a) and the alternative (17b) are not contextually equivalent. Let me now turn to the more delicate assumption (11), that the exhaustivity operator is mandatory. In plain cases, implicatures are well known to be context dependent. For instance, sentence (17a) triggers the scalar implicature that the alternative (17b) is false (namely that John did only some of the homework) in the context of the background question "How much homework did John do?". But that implicature is much weaker and perhaps unattested in the case of the background question "Who did some of the homework?". If the presence/absence of the implicature were due to the presence/absence of the exhaustivity operator as conjectured in (18), then implicatures' context dependence would contradict assumption (11) that the exhaustivity operator is mandatory.

(18) we get the implicature that (17b) is false $\Longleftrightarrow \quad \mathrm{EXH}_{\mathscr{R}}$ is present at LF we do not get the implicature that (17b) is false $\Longleftrightarrow \quad \mathrm{EXH}_{\mathscr{R}}$ is absent at LF

Yet, there is an alternative way to account for the context sensitivity of the implicature associated with the alternative (17b): the exhaustivity operator is always present at LF; yet, in certain contexts the alternative (17b) is not relevant (namely it does not belong to $\mathscr{R}$ ) and thus does not get negated by the exhaustivity operator. This alternative account (19) is based on an insight from Fox \& Spector (2008). From this perspective, implicature cancellation is just a special case of run of the mill contextual domain restriction. By (19), assumption (11), that the exhaustivity operator is mandatory, is compatible with implicatures' context dependence

(19) we get the implicature that (17b) is false $\Longleftrightarrow \quad(17 b) \in \mathscr{R}$ we do not get the implicature that (17b) is false $\Longleftrightarrow \quad(17 \mathrm{~b}) \notin \mathscr{R}$

In conclusion, the crucial difference between plain and odd cases is as follows: in plain cases, the alternative might not be relevant and thus the implicature is optional; while in odd cases, the alternative is necessarily relevant (because contextually equivalent to the prejacent) and thus the corresponding implicature is mandatory.

\section{Some new facts about oddness in downward entailing contexts}

This section pursues the analysis of oddness further, moving from the unembedded cases (1)-(3) considered so far to cases of embedding in downward entailing (DE) contexts. 
The data Here is a first set of data on oddness in DE contexts, using the DE context provided by the restrictor of a universal quantifier. ${ }^{3}$

a. \#Le italiane vengono da un paese caldo.

The Italians-FEM come from a country warm

'Italian women come from a warm country'

b. Gli italiani vengono da un paese caldo.

The Italians-MASC come from a country warm

'Italians come from a warm country'

(21) Context: Every year, the dean has to decide: if the college has made enough profit that year, he gives a pay raise to every professor who has assigned an A to at least some of his students; if there is not enough money, then no one gets a pay raise.

a. ... \#This year, every professor who assigned an A to all of his students got a pay raise.

b. ... This year, every professor who assigned an A to some of his students got a pay raise.

These sentences fit into the scheme (22a), where $R$ is the restrictor of the universal operator and $P$ its nuclear scope. The two sentences in each pair only differ because of the restrictor of the universal quantifier. Thus, I will abbreviate (22a) as in (22b).

a. for every $x$ such that $R(x)$, it is the case that $P(x)$.

b. every $_{x} R(x)$.

The restrictors of the universal quantifiers in the two sentences (20a) and (20b) are Strong and Weak in (23), respectively. Here, I am assuming that masculine morphology is semantically vacuous, after Sauerland (2004). The restrictors for the two sentences (21a) and (21b) are Strong and Weak in (24). The names Strong/Weak reflect the fact that in both cases Strong $(x)$ asymmetrically entails Weak $(x)$.

$$
\begin{aligned}
& \text { Strong }(x)=x \text { is an Italian woman. } \\
& \text { Weak }(x)=x \text { is an Italian (man or woman). } \\
& \text { Strong }(x)=x \text { gave an A to all of his students. } \\
& \text { Weak }(x)=x \text { gave an A to some of his students. }
\end{aligned}
$$

With notation (22)-(24), the pattern of oddness in this first set of data (20)-(21) can be schematized as (25).

$$
\begin{gathered}
\text { \#every(Strong); } \\
\sqrt{ } \text { every(Weak). }
\end{gathered}
$$

3 For the case of (20), I assume that plural definites (and generics) are universal operators. 
Another argument for embedded scalar implicatures

A second set of data is provided in (26)-(27). Note that sentences (27) are identical to those in (21), the only difference being the background knowledge considered.

(26) Context: in a society where all children inherit the last name of their father.

a. Every father whose children have a funny last name must pay a fine.

b. \#Every father some of whose children have a funny last name must pay a fine.

(27) Context: In this department, every professor assigns the same grade (a different one every year) to all of his students.

a. This year, every professor who assigned an A to all of his students got a pay raise.

b. \#This year, every professor who assigned an A to some of his students got a pay raise.

The restrictors of the universal quantifiers in the two sentences (26a) and (26b) are Strong and Weak in (28), respectively. The restrictors for the two sentences (27a) and (27b) are Strong and Weak in (24), repeated in (29). Again, Strong (x) (logically) asymmetrically entails Weak $(x)$ in both cases.

(28) Strong $(x)=x$ is a father all of whose children have a long last name.

Weak $(x)=x$ is a father some of whose children have a long last name.

Strong $(x)=x$ gave an A to all of his students.

Weak $(x)=x$ gave an A to some of his students.

With notation (22) and (28)-(29), the pattern of oddness (26)-(27) can be schematized as (30). Interestingly, the two patterns (25) and (30) are the opposite of each other.

$$
\begin{aligned}
& \sqrt{ } \text { every }(\text { Strong }) \\
& \text { \#every }(\text { Weak })
\end{aligned}
$$

So far, I have used the restrictor of universal quantifiers as the relevant DE context. But the same pattern holds for other DE contexts. In (31) and (32), I consider the variants of (21) and (27), with every replaced by no (and furthermore some replaced by disjunction to circumvent PPIness of the former). See Magri 2009b for discussion of further DE contexts.

(31) Context: Every year, the dean has to decide: if the college has made enough profit that year, he gives a pay raise to every professor who has taught a graduate or an undergraduate class; if there is not enough money, then no one gets a pay raise.

a. This year, no professor who taught a graduate or an undergraduate class got a pay raise. 
b. \#This year, no professor who taught a graduate and an undergraduate class got a pay raise.

(32) Context: In this department, every professor teaches both a graduate and an undergraduate class in the same field of linguistics.

a. \#This year, no professor who taught graduate or undergraduate Semantics got a pay raise

b. This year, no professor who taught graduate and undergraduate Semantics got a pay raise.

The two sentences in each pair (20)-(21) and (26)-(27) are contextually equivalent and only differ w.r.t. the restrictor of the universal quantifier, Strong vs. Weak. I conclude that the data on oddness in DE contexts split into two opposite patterns (25) and (30). The contrast between (21) and (27) is particularly puzzling: the same two sentences give rise to the opposite patterns of oddness (25) and (30), depending on the common knowledge considered.

A generalization about oddness in DE contexts What is the relevant difference between the two opposite patterns (25) and (30)? I submit that the relevant difference is (33). Pattern (25) is characterized by the fact that the two competing restrictors Weak and Strong in (23)-(24) are not contextually equivalent (although the matrix sentences are): Weak and Strong in (23) are not equivalent because of the existence of Italian men; and Weak and Strong in (24) are not equivalent because the context set up in (21) does not in any way entail that professors that gave an A to some of their students also gave an A to all of them. Pattern (30) is instead characterized by the fact that the two competing restrictors Weak and Strong in (28)-(29) are indeed contextually equivalent: Weak and Strong in (28) are contextually equivalent because there is no father whose children have different last names; and Weak and Strong in (29) are contextually equivalent because the context set up in (27) ensures that professors give the same grade to all of their students.

(33) a. In the case of (25), Weak and Strong are not contextually equivalent.

b. In the case of (30), Weak and Strong are indeed contextually equivalent.

By putting together (25), (30) and (33), we get the generalization (34). Here, I have stated the generalization only for the special DE context provided by the restrictor of a universal quantifier. But the facts in (31)-(32) suggest that the generalization extends to any DE context; see Magri 2009b for further discussion.

(34) Assume that the matrix sentences every(Strong) and every(Weak) are contextually equivalent. Then:

a. if the embedded Strong and Weak are not contextually equivalent, then: 
Another argument for embedded scalar implicatures

i. \#every (Strong)

$={ }^{‘}$ Every Italian woman...'

ii. $\sqrt{ }$ every $($ Weak $)$

$=$ 'Every Italian...'

This I call the Italian Women (IW) pattern.

b. if the embedded Strong and Weak are contextually equivalent, then:

i. $\sqrt{ }$ every $($ Strong $)$ $=$ 'Every father whose children...'

ii. \#every $($ Weak $)$ $=$ 'Every father some of whose children...'

This I call the Italian Fathers (IF) pattern.

Is there a way to extend Hawkins' reasoning (5) to account for generalization (34)? Hawkins' reasoning works prima facie straightforwardly for the IW pattern in (34a), according to which the logically stronger sentence every(Weak) is fine and the logically weaker sentence every(Strong) is odd. But Hawkins' reasoning miserably fails on the IF pattern in (34b), according to which the logically stronger sentence every (Weak) is odd and the logically weaker sentence every(Strong) is fine! It is useful to understand in full detail how Hawkins' reasoning fails to account for the IF pattern. In fact, it turns out that generalization (34) raises two different challenges for Hawkins' reasoning, discussed in the rest of this section.

The first challenge raised by generalization (34). Consider the odd sentence (35a) of the IW pattern together with the fine sentence (35b) of the IF pattern.
a. \# $\underbrace{\text { Italian women }}_{\text {Strong }}$ come from a warm country.

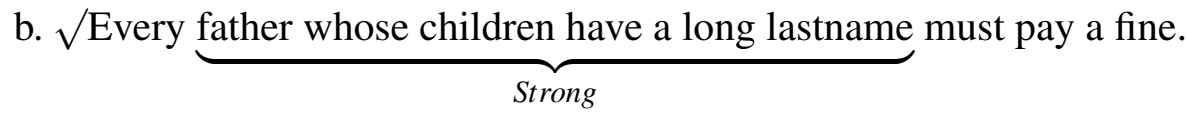

As shown in (36), Hawkins' reasoning accounts for the oddness of the odd sentence (35a) of the IW pattern through a mismatching implicature, just as it did for the unembedded cases (1)-(3) above: by (11), the LF comes with a matrix exhaustivity operator, as in (36a); by (10), the implicature is mandatory because of the contextual equivalence between the alternative and the prejacent, as in (36b); the sentence thus means a contextual contradiction, as stated in (36c).

$$
\begin{aligned}
& {[[\# \text { Italian women come from a warm country }]]=} \\
& \stackrel{(a)}{=} \operatorname{EXH} \mathscr{R}(\underbrace{\text { All Italian women c.f.w.c. }}_{\text {every }(\text { Strong })}) \\
& \stackrel{(b)}{=} \underbrace{\text { All Italian women c.f.w.c. }}_{\text {every }(\text { Strong })} \text { and not } \underbrace{\text { all Italians c.f.w.c. }}_{\text {every }(\text { Weak })} \\
& \stackrel{(c)}{=} \text { contextual contradiction }
\end{aligned}
$$


Yet, by parity of reasoning, Hawkins' reasoning derives a mismatching implicature also for the fine sentence (35b) of the IF pattern, as shown in (37).

$$
\begin{aligned}
& {[[\sqrt{ } \text { Every father all of whose children....) }]=} \\
& =\operatorname{EXH}_{\mathscr{R}}(\underbrace{\text { Every father all of whose children... }}_{\text {every }(\text { Strong })}) \\
& =\underbrace{\text { Every father all of whose.... }}_{\text {every }(\text { Strong })} \text { and not } \underbrace{\text { every father some of whose... }}_{\text {every }(\text { Weak })} \\
& =\text { contextual contradiction }
\end{aligned}
$$

Thus, the first challenge raised by generalization (34) for Hawkins' reasoning is as follows: I need to protect the fine sentence (35b) of the IF pattern from the same mismatching implicature used to kill the odd sentence (35a) of the IW pattern.

The second challenge raised by generalization (34). Consider now the good sentence (38a) of the IW pattern together with the odd sentence (38b) of the IF pattern.
a. $\sqrt{ } \underbrace{\text { Italians }}_{\text {Strong }}$ come from a warm country.
b. \#Every father some of whose children have a long lastname must pay a fine.

As shown in (39), Hawkins' reasoning accounts for the fine status of the fine sentence (38a) of the IW pattern because there is no mismatching implicature: by (11), again the LF comes with a matrix exhaustivity operator, as in (39a); but no implicature arises because the prejacent is logically strongest, as in (39b).

$$
\begin{aligned}
{[[\sqrt{ } \text { Italians come from a warm country }] \rrbracket} & \stackrel{(a)}{=} \operatorname{EXH} \mathscr{R}(\underbrace{\text { All Italians c.f.w.c. }}_{\text {every(Weak })}) \\
& \stackrel{(b)}{=} \text { all Italians c.f.w.c. }
\end{aligned}
$$

Yet, by parity of reasoning, I get no mismatching implicature also for the odd sentence (35b) of the IF pattern, as shown in (40).

$$
\begin{aligned}
{[[\sqrt{ } \text { Every father some of whose... }]] } & =\operatorname{EXH}_{\mathscr{R}}(\underbrace{\text { Every father some of whose... }}_{\text {every }(\text { Weak })}) \\
& =\text { Every father some of whose... }
\end{aligned}
$$

Thus, the second challenge raised by generalization (34) for Hawkins' reasoning is as follows: I need to cook up a mismatching implicature for the odd sentence (38b) of the IF pattern, and protect from it the fine sentence (38a) of the IW pattern. 
Another argument for embedded scalar implicatures

\section{An account for oddness in DE contexts based on embedded implicatures}

In this section, I argue that both challenges outlined at the end of the preceding section can be met by running Hawkins' reasoning both at the matrix and at the embedded level. As Hawkins' reasoning relies on scalar implicatures, the proposal thus bears on the existence of embedded scalar implicatures.

Mandatory embedded exhaustivity operators. I assumed in (11) that the exhaustivity operator must mandatorily appear at LF. But appear where? Well, for the unembedded cases (1a)-(3a), it was enough that the exhaustivity operator be mandatory at matrix scope. But what is special about matrix scope? Nothing. Thus, I refine my initial assumption (11) as in (41).

(41) The exhaustivity operator $\mathrm{EXH}_{\mathscr{R}}$ is mandatory at every scope site.

Assumption (41) says, in particular, that there are embedded exhaustivity operators in DE contexts. Thus, the proper LF of (42a) is (42b), with a matrix exhaustivity operator and another one embedded in the restrictor of the universal quantifier. ${ }^{4,5}$

(42) a. Everyone who did some of the homework will get an A.

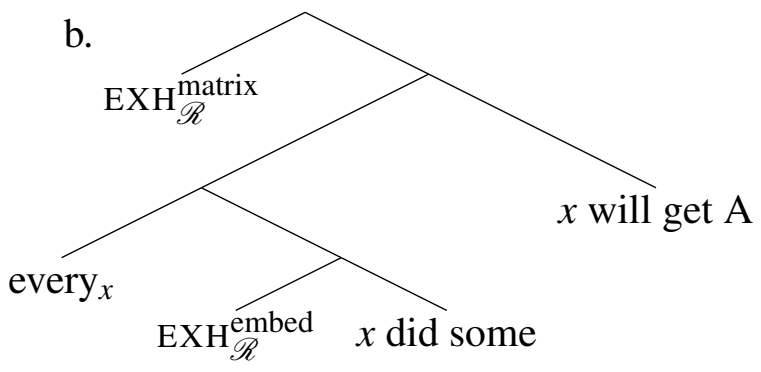

Assumption (41) is my only refinement of the theory sketched in section 2 . The other assumptions stay the same: the basic semantics (6)-(7) of the exhaustivity operator; the blindness assumption (9); the axioms (10) on relevance.

Nothing changes for plain cases. By my initial assumption (11), the exhaustivity operator is mandatory at matrix level. In section 2 , I have defended this assumption

4 In (6), I have construed the exhaustivity operator as a propositional operator. In order to put an exhaustivity operator in the restrictor of 'every' in (42), I thus need the restrictor to have type $t$. For this reason, I let the quantifier bind a variable $x$ that saturates the restrictor. Alternatively, I could redefine the exhaustivity operator for arbitrary types "that end in $t$ ", and stick to the more standard assumption that the type of the restrictor is $\langle e, t\rangle$.

5 Of course, assumption (41) also forces an exhaustivity operator in the nuclear scope of the universal quantifier. But I ignore that third instance of the exhaustivity operator here, as the nuclear scope contains no scalar item. 
as follows. Of course, there are plain cases that trigger no matrix implicature. Aren't those counterexamples to assumption (11)?! Well no: there is no implicature not because there is no exhaustivity operator but because relevance has trimmed from its domain the corresponding alternative. This logic carries over to the extension from (11) to (41), from matrix to arbitrary scope, as follows. There seem to be no implicatures in DE contexts in plain cases (without focus on the scalar item): for instance, sentence (42a) can hardly be interpreted as saying that whoever did only some of the homework got an A. Aren't those cases counterexamples to assumption (41), that in particular forces an exhaustivity operator in DE contexts, as in (42b)?! Well no, as I can reason analogously to the unembedded case, as follows. Following Fox \& Spector (2008), assume that the lack of implicatures in DE contexts in plain cases is due to a constraint such as (43).

(43) An occurrence of the exhaustivity operator is ungrammatical if it leads to a globally weaker meaning.

By (43), the embedded exhaustivity operator in LF (42b) would be ungrammatical if it did negate the universally quantified alternative. In order for the LF (42b) to cope with (43), it is thus crucial that relevance trims from the domain of the embedded exhaustivity operator the universally quantified alternative, just as it did for those unembedded plain cases where the implicature was unattested, as in (19). ${ }^{6}$

Main claim of the paper. The two challenges raised by generalization (34) for Hawkins' reasoning discussed at the end of section 3 were due to the fact that I was applying Hawkins' reasoning only at the matrix level. Yet, it turns out that:

(44) Both challenges are solved by assuming (41): that the exhaustivity operator is mandatory at every scope site and that Hawkins' reasoning therefore also applies in embedded positions.

I prove claim (44) in the Appendix (section 5). Let me present here the idea informally. Consider first the "easy" IW pattern, repeated in (45). This is the case where the two restrictors Strong and Weak are not contextually equivalent. The idea of the account is straightforward: since Weak and Strong are not contextually equivalent, nothing forces the embedded implicature; thus, the exhaustivity operator

6 Yet, there are admittedly two crucial differences between the unembedded case considered in section 2 and the embedded case considered here. One difference is that in the embedded case, relevance is predicated of an open formula like $x$ did some, with a free variable $x$. This requires the intuitive notion of relevance to be formalized carefully. Another difference is that in the embedded case it is the grammatical condition (43) that forces relevance to trim an alternative, rather than a property of the context of utterance. The proposal developed here thus predicts that a sentence such as (42) triggers certain presuppositions on relevance. A close investigation of this prediction is deferred to future work. 
Another argument for embedded scalar implicatures

embedded in the restrictor of the universal quantifier does nothing; in other words, the pattern of oddness in the case where Weak and Strong are not contextually equivalent is determined by the matrix exhaustivity operator; hence, the matrix logically weaker sentence $(45 \mathrm{a})=$ every (Strong) sounds odd while the matrix logically stronger sentence $(45 \mathrm{~b})=$ every $($ Weak $)$ sounds fine.

$\begin{array}{ll}\text { a. \# } \underbrace{\text { Italian women }}_{\text {Strong }} \text { come from a warm country. } & \text { \#every }(\text { Strong) } \\ \text { b. } \sqrt{ } \underbrace{\text { Italians }}_{\text {Weak }} \text { come from a warm country. } & \sqrt{ } \text { every }(\text { Weak })\end{array}$ Consider next the "hard" IF pattern, repeated in (46). This is the case where the two restrictors Strong and Weak are indeed contextually equivalent. The idea of the account is as follows: since Strong and Weak are contextually equivalent, then the embedded implicature is mandatory; thus, 'every $(\text { Weak })^{\prime}=(46 \mathrm{~b})$ is odd because the mandatory embedded implicature screws up the matrix prejacent; and 'every $($ Strong $) '=(46 a)$ is fine because the mandatory embedded implicature screws up the potentially harmful matrix implicature.

a. $\sqrt{ }$ Every $\underbrace{\text { father all of whose children... }}_{\text {Strong }}$ will pay a fine. $\quad \sqrt{\text { every }(\text { Strong })}$
b. \#Every $\underbrace{\text { father some of whose children... }}_{\text {Weak }}$ will pay a fine. $\quad$ \#every $($ Weak $)$

Turning the perspective of claim (44) upside down, I conclude with the main point of this paper: the pattern of oddness in DE contexts schematized in generalization (34) provides new evidence for embedded scalar implicatures - actually, embedded where you would least expect them, namely in DE contexts!

\section{Appendix: proof of claim (44)}

On the first challenge raised by generalization (34). Consider again the odd sentence (47a) of the IW pattern and the fine sentence (47b) of the IF pattern.
a. \# $\underbrace{\text { Italian women }}_{\text {Strong }}$ come from a warm country.
b. $\sqrt{ }$ Every father whose children have a long lastname must pay a fine.

By assumption (41) that the exhaustivity operator is mandatory at every scope site, the LF of sentences (47) is (48), with both a matrix and an embedded exhaustivity operator. Here, Strong is either (23a) or (28a), respectively. 
Giorgio Magri

(48)

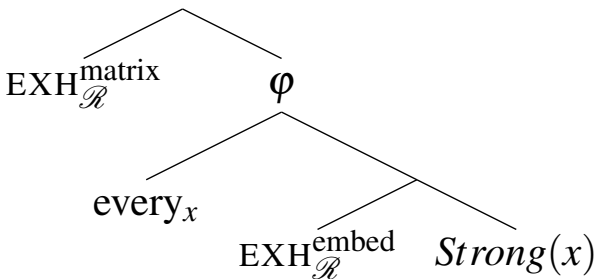

Since Weak is not excludable w.r.t. Strong, the embedded exhaustivity operator does nothing and the matrix prejacent $\varphi$ ends up with the truth conditions (49).

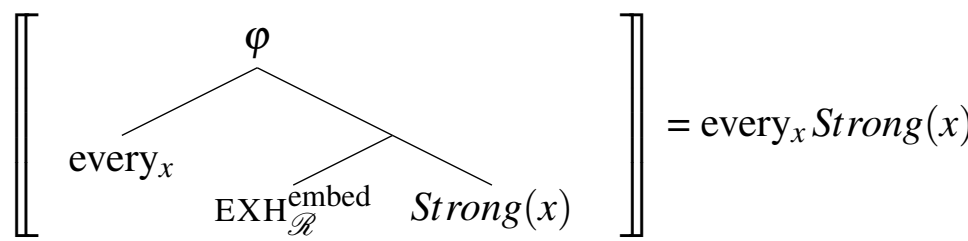

The matrix alternative is $\psi$ obtained by replacing Strong with Weak, as defined in (23b) and (28b). Depending on whether Strong is relevant or not, we get one of the two truth conditions in (50). In either case, the alternative $\psi$ is logically excludable w.r.t. the prejacent $\varphi$.

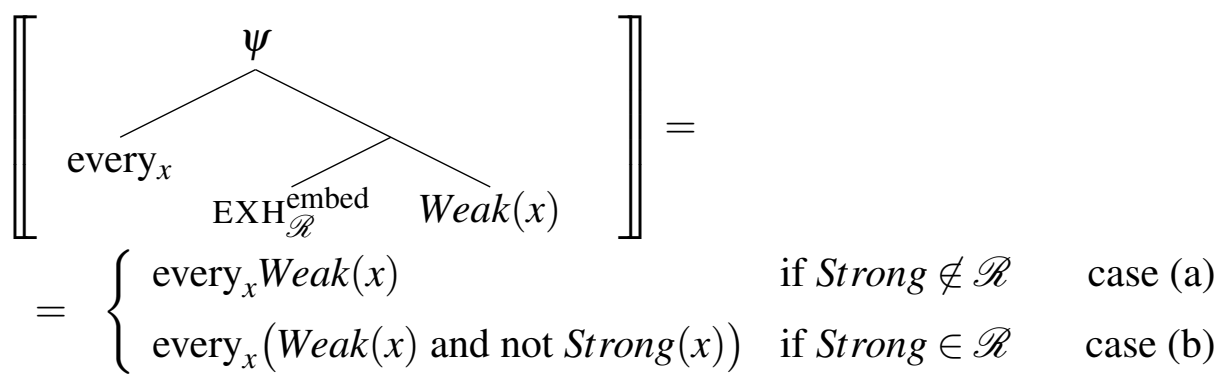

Consider first the case of (47a), characterized by the fact that Strong and Weak are not contextually equivalent. No matter whether the alternative $\psi$ ends up with the truth conditions (50a) or (50b), it is contextually equivalent to the prejacent $\varphi$ in (49). By assumptions (10), the matrix implicature is thus mandatory. In conclusion, we derive the truth conditions $[[(47 \mathrm{a})]]=\varphi \wedge \neg \psi$, that are a contextual contradiction. ${ }^{7}$

7 I am assuming a framework where there are exhaustivity operators both in the prejacents as well as in the alternatives, as in (49) and (50), raising the issue of whether conditions such as (43) apply only to the exhaustivity operators in the prejacents or also in the alternatives. If (43) applies also to the alternative (50), then it rules out the option of truth conditions (50b) for the alternative $\psi$, leaving open only the option of truth conditions (50a). If instead (43) does not apply also to the alternative (50), then both truth conditions (50a) and (50b) are in principle available for the alternative $\psi$. In the latter case, it is crucial to choose the definition of excludable alternatives in (7b) rather than the one in (7a). In fact, if the truth conditions of the alternative $\psi$ are (50b), then $\psi$ does not logically asymmetrically entail the prejacent $\varphi$ in (49). Thus, if I had assumed the definition (7a) of the set of excludable alternatives, the matrix exhaustivity operator would have ended up with no alternatives, with the result that no mismatching implicature would have been derived. This difficulty does not 
Another argument for embedded scalar implicatures

Consider next the case of (47b), characterized by the fact that Strong and Weak are indeed contextually equivalent. By assumption (10), the embedded implicature is forced in place and the truth conditions of the alternative $\psi$ are therefore $(50 \mathrm{~b})$. Because of the contextual equivalence between Strong and Weak, the alternative $\psi=$ every $_{x}($ Weak $(x)$ and not Strong $(x))$ is thus either a tautology or a presupposition failure. In either case, it is not contextually equivalent to the prejacent $\varphi$ in (49). Thus, no matrix implicature is predicted, and we thus end up with the fine truth conditions $[[(47 \mathrm{~b})]]=\varphi^{8}{ }^{8}$

On the second challenge raised by generalization (34). Consider again the good sentence (51a) of the IW pattern and the odd sentence (51b) of the IF pattern.

a. $\sqrt{ } \underbrace{\text { Italians }}_{\text {Weak }}$ come from a warm country.

b. \#Every father some of whose children have a long lastname must pay a fine.

Weak

By assumption (41) that the exhaustivity operator is mandatory at every scope site, the LF of sentences (51) is (52), with both a matrix and an embedded exhaustivity operator. Here, Weak is either (23b) or (28b), respectively.

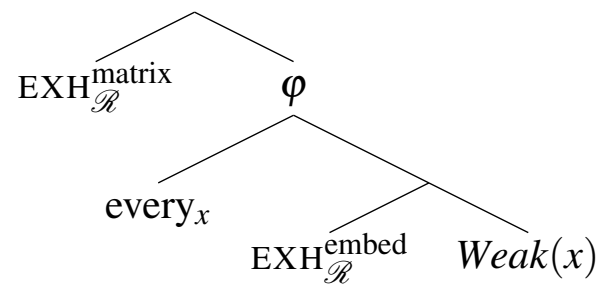

Depending on whether Strong is relevant or not, the prejacent $\varphi$ has one of the two truth conditions in (53).

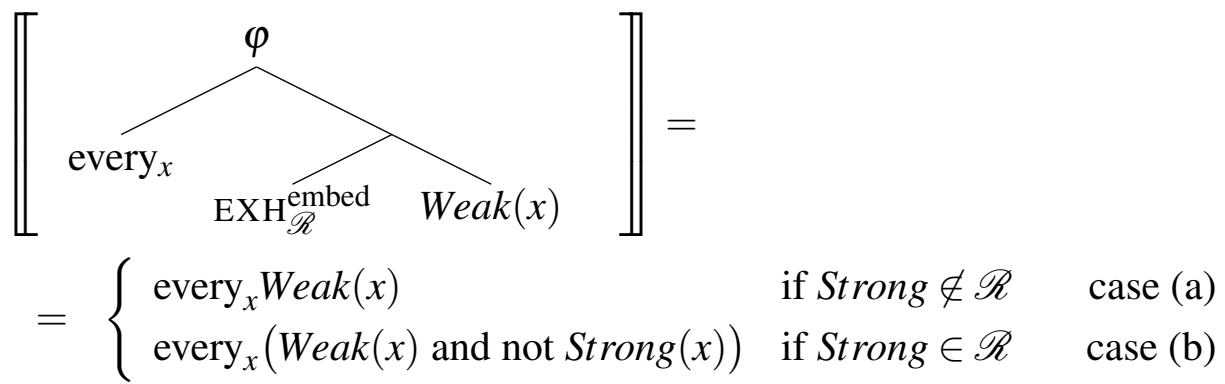

arise with the alternative definition (7b) of the set of excludable alternatives: in this case, no matter whether the alternative $\psi$ ends up with the truth conditions (50a) or (50b), its negation is logically compatible with the prejacent $\varphi$ in (49) and thus counts as a matrix excludable alternative.

8 Another option is of course that the alternative $\psi=$ every $_{x}($ Weak $(x)$ and not Strong $(x))$ in (50b) is ruled out by (43), provided that the latter applies also to the exhaustivity operators in the alternatives. 
The matrix alternative is $\psi$ obtained by replacing Weak by Strong, as defined in (23a) or (28a). Since Weak is not excludable w.r.t. Strong, the embedded EXH in the alternative $\psi$ does nothing. Thus, $\psi$ ends up with the plain truth conditions (54).

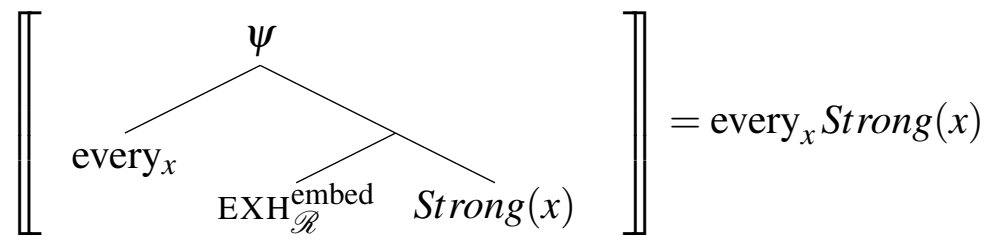

Consider first the case of (51a), where the two restrictors Strong and Weak are not contextually equivalent. Thus, I am free to assume Strong is not relevant, without violating (10). In this case, the embedded exhaustivity operator in the matrix prejacent $\varphi$ does nothing and the matrix prejacent $\varphi$ ends up with the plain truth conditions (53a). Of course, the alternative $\psi$ in (54) is not excludable w.r.t. the prejacent $\varphi$, and thus also the matrix exhaustivity operator does nothing. We thus derive the plain truth conditions $[[(51 \mathrm{a})]]=$ every $_{x} \operatorname{Weak}(x)$, that are of course not a contextual contradiction. Consider next the case of (51b), where the two restrictors Strong and Weak are indeed contextually equivalent. By axioms (10) on relevance, Strong is relevant, because contextually equivalent to the embedded prejacent Weak. Thus, the truth conditions of the matrix prejacent are (53b). No matter what the matrix exhaustivity operator does, the matrix prejacent is thus ruled out, either because it is a presupposition failure or a tautology (as the restrictor of 'every' is contextually empty) or because it violates Fox \& Spector's (2008) condition (43).

\section{References}

Chemla, Emmanuel \& Benjamin Spector. 2009. Experimental evidence for embedded implicatures. Ms. IJN \& LSCP.

Chierchia, Gennaro. 2004. Scalar implicatures, polarity phenomena and the syntax/pragmatics interface. In A. Belletti (ed.), Structures and Beyond, Oxford: Oxford University Press.

Chierchia, Gennaro, Danny Fox \& Benjamin Spector. to appear. The Grammatical View of Scalar Implicatures and the Relationship between Semantics and Pragmatics. In Paul Portner, Claudia Maienborn \& Klaus von Heusinger (eds.), Handbook of Semantics, Berlin, New York: Mouton de Gruyter.

Fox, Danny. 2007. Free Choice and the Theory of Scalar Implicatures. In U. Sauerland \& P. Stateva (eds.), Presupposition and Implicature in Compositional Semantics, 71-120. New York: Palgrave Macmillan.

Fox, Danny \& Martin Hackl. 2006. The Universal Density of Measurement. Linguistics and Philosophy 29(5). 537-586. doi:10.1007/s10988-006-9004-4.

Fox, Danny \& Roni Katzir. 2009. Alternatives for implicature and focus. In Paul 
Another argument for embedded scalar implicatures

Égré \& Giorgio Magri (eds.), Presuppositions and Implicatures. Proceedings of the MIT-Paris Workshop, 101-110. Cambridge, MA: MIT Working Papers in Linguistics, volume 60.

Fox, Danny \& Benjamin Spector. 2008. Economy and Embedded Exhaustification. MIT presentation on May 30, 2008.

Geurts, Bart \& Nausicaa Pouscoulous. 2009. Embedded Implicatures?!? Semantics and Pragmatics 2(4). 1-34. doi:10.3765/sp.2.4.

Grice, Paul. 1975. Logic and Conversation. In P. Cole \& J. Morgan (eds.), Syntax and Semantics 3: Speech Acts, 41-58. New York: Academic: Academic Press.

Hawkins, John A. 1991. On (in)definite articles: implicatures and (un)grammaticality prediction. Journal of Linguistics $27.405-442$. doi:10.1017/S0022226700012731.

Heim, Irene. 1991. Artikel und Definitheit. In A. von Stechow \& D. Wunderlich (eds.), Semantik: Ein internationales Handbuch der zeitgenössischen Forschung, 487-535. De Gruyter: Berlin.

Magri, Giorgio. 2009a. A Theory of individual Level Predicates Based on Blind Mandatory Scalar Implicatures. Natural Language Semantics 17(3). 245-297. doi:10.1007/s11050-009-9042-x.

Magri, Giorgio. 2009b. A theory of individual-level predicates based on blind mandatory scalar implicatures. Constraint promotion for Optimality Theory: MIT dissertation.

Percus, Orin. 2006. Antipresuppositions. In A. Ueyama (ed.), Theoretical and Empirical Studies of Reference and Anaphora: toward the Establishment of Generative Grammar as an Empirical Science, Report of the Grant-in-Aid for Scientific Research (B), Project No. 15320052, Japan Society for the Promotion of Science, 52-73.

Russell, Benjamin. 2006. Against Grammatical Computation of Scalar Implicatures. Journal of Semantics 23(4). 361-382. doi:10.1093/jos/ffl008.

Sauerland, Uli. 2004. A Comprehensive Semantics for Agreement. Paper presented at the Phi-Workshop, McGill University, Montreal, Canada.

Sauerland, Uli. 2008. Implicated Presuppositions. In A Steube (ed.), Sentence and Context. Language, Context and Cognition, -. Berlin, Germany: Mouton de Gruyter.

Schlenker, Philippe. 2006. Maximize Presupposition and Gricean Reasoning. Manuscript, UCLA and Institute Jean-Nicod.

Giorgio Magri

IJN, DEC, ENS, CNRS

magrigrg@gmail.com 